\title{
Diseño asistido por computador
}

(1) Oswaldo Rojas Lazo

(2) Luis Rojas Rojas

\section{RESUMEN}

El diseño es una actividad que se proyecta hacia la solución de problemas planteados por el ser humano en su adaptación al medio que lo rodea, para la satisfacción de sus necesidades, para lo cual utiliza recursos como la tecnología CAD/CAE/CAM. Estas tecnologías se vienen aplicando a través de los métodos de la ingeniería concurrente. La técnica más desarrollada en la ingeniería asistida por computador (CAE), es la aplicación de los análisis por elementos finitos (FEA), que con la mejora de los equipos de cómputo se ha convertido en técnicas accesibles para todos los usuarios. Estas técnicas son usadas industrialmente desde el diseño hasta la fabricación consiguiendo optimizar costos, calidad, tiempo, seguridad, etc.

Palabras Clave: Diseño asistido por computador, CAD, CAE.

\section{COMPUTER AIDED DESIGN ABSTRACT}

Design is an activity that is projected to the problem solutions. These problems are analyzed by the human being when they try to adapt themselves to their surrounding environment, to satisfy their needs, using resources like CAD/CAE/CAM technologies. These technologies are currently applied through engineering concurrent methods. The most developed technique in the compute assisted engineering, is the application of the finite element analysis, which with the improvement of computer equipment, ithas become in accessible techniques for all users. From design thru manufacturing, these techniques are used by the industries, having optimized costs, quality, time, safety, and so on.

Key words: Computer aided design, CAD, CAE.

\section{INTRODUCCIÓN}

Para poder entender los conceptos que se desarrollarán en la presente publicación es necesario conocer las siguientes siglas:

CAD (Computer Aided Design): Diseño asistido por computador.

Cad (Computer aided drafting): Dibujo asistido por computador.

CADD (Computer Aided Design and Drafting): Diseño y dibujo asistido por computadora.

CAE (Computer Aided Engineering): Ingeniería asistida por computador. CAM (Computer Aided Manufacturing): Manufactura asistida por computador. CIM (Computer Integrated Manufacturing) Manufactura integrada por computador. FEA (Finite Element Analysis): Análisis por elementos finitos.

En publicación anterior de esta revista (Vol. 8, Nro. 1, 2005), se desarrolló el artículo sobre el Dibujo Asistido por Computador, el presente artículo es una continuación.

El avance vertiginoso del software y hardware, en estos últimos años ha modificado la forma de entender el concepto de CAD, actualmente se entiende como la integración del diseño y del análisis (Cad unida al CAE). La técnica CAE necesita de una gran potencia de cálculo de los computadores, lo cual implica una memoria RAM considerable, velocidad de proceso y una calidad de exhibición de los resultados; estas características se vienen consiguiendo con los nuevos computadores a precios aceptables para nuestro medio. Esto ha permitido que los profesionales relacionados a estas tecnologías mejoren su productividad, calidad y oportunidad, de manera que puedan dedicar un mayor tiempo en la mejora de los diseños.

Desde que en 1963 Ivan Sutherland del MIT (Massachussets Institute of Technology) publicó su tesis doctoral sobre graficas interactivas por computadora definiendo la topología del objeto (sistema Sketchpad - SUTH63) la tecnología CAD se ha desarrollado con la limitante del tiempo de proceso. Una de las herramientas de análisis más estudiado y aplicado son los cálculos con elementos finitos que permiten predecir con gran precisión y simplicidad los esfuerzos y deformaciones que soportará internamente una pieza o conjunto de piezas al ser sometidas a un sistema de cargas.

La aplicación del software CAD en la ingeniería abarca la elaboración de cuadros sinópticos, diagramas de diversos tipos, gráficos estadísticos, representación normalizada de piezas para su diseño y fabricación, representación tridimensional de modelos dinámicos en multimedia, análisis con elementos finitos, aplicaciones en realidad virtual, robótica, etc.

(1) Ingeniero Industrial. Profesor del Departamento de Diseño y Tecnología Industrial, UNMSM E-mail: orojasl@unmsm.edu.pe

(2) Bachiller en Ingeniería Mecánica

E-mail: jorger2@hotmail.com 
Los software CAD pueden ser usados de dos maneras generales, a través de lenguajes de programación y de paquetes aplicativos. El desarrollo a través de lenguajes de programación abiertos implica un amplio dominio, conocimiento de las tecnologías de exhibición, manejo del análisis matemático, geométrico y vectorial (software abiertos más usados: Java y Visual Basic); en cambio el uso de paquetes aplicativos debido a su amplio desarrollo acelerado, su especialización en los diferentes campos de aplicación, su diseño de arquitectura abierta y su facilidad de uso han permitido su rápida aceptación y adopción.

El CAD es una técnica de análisis, una manera de crear un modelo del comportamiento de un producto aun antes de que se haya construido. Los dibujos en papel pueden no ser necesarios en la fase del diseño.

Las características generales que deben tener el software CAD/CAE son:

- Simulaciones dinámicas con características especiales de visualización de procesos y resultados (representaciones foto realistas, tabulaciones, diagramas, giros, sonido, etc.).

- Capacidad del software de generar soluciones óptimas según los tipos de aplicación.

- Desarrollo de sistemas virtuales dentro de un entorno, permitiendo en muchos casos eliminar los prototipos físicos.

- Ingeniería concurrente on-line (trabajo multidisciplinario vía red, con niveles de acceso y con geoprocesamiento referenciado).

- Arquitectura abierta del software (posibilidad de personalizar y generar programas complementarios - "glue functions").

- Ingeniería inversa (obtener un modelo CAD a partir del escaneado tridimensional de una pieza real).

- Intercambio estandarizado de formatos de archivos para el trabajo multiplataforma (run anywhere).

- Pantalla de trabajo (workspace) compartidos con diferentes aplicaciones y programas adicionales (plug-ins).

La incorporación de la computada es en la producción el elemento puente que está permitiendo lograr la automatización integral de los procesos industriales así como una integración tecnológica de sus áreas. Es así que los software profesionales están tendiendo a integrase bajo un gran sistema CAD/ CAM/CAE que ha puesto de relieve la importancia de automatizar informáticamente cualquier proceso industrial desde el diseño hasta la fabricación. Esta informatización incidirá de forma directa sobre el proceso de varias formas:

- Reducción de tiempos (time to market) y mayor sencillez en la etapa de diseño.
- Seguridad de un correcto funcionamiento debido a un simulado del prototipo.

- Fácil integración en una cadena de fabricación y mejora en la gestión del proyecto.

- Obtención de un producto económico, de óptima calidad y menor tiempo.

El avance de la ingeniería se viene dando fundamentalmente por los nuevos y/o mejora de los materiales, equipos y herramientas de trabajo, descubrimiento y aplicación de nuevos conceptos. Estos avances han sido favorecidos por el desarrollo de las tecnologías CAD.

\section{PROCESO DE DISEÑO DE INGENIERÍA}

El diseño en ingeniería es el proceso de concebir ideas en el desarrollo de la solución de un problema tecnológico, para lo cual usa conocimientos, recursos y productos existentes para satisfacer una necesidad o resolver un problema.

El diseño puede dividirse en dos grandes categorías: diseño de productos y diseño de sistemas o procesos. A medida que se desarrolla el diseño de un producto o proceso, el equipo de diseño aplica principios de ingeniería, toma en cuenta las restricciones de presupuesto, funcionalidad, legales, psicológicos y sociales para lo cual requiere información de áreas como las necesidades del cliente, materiales, capital, energía, requerimientos de tiempo, habilidades humanas, etc.

Las gráficas son importantes en el proceso de diseño, se utiliza para visualizar soluciones posibles y documentar las ideas, incluyen el dibujo de las piezas, curvaturas, descripciones de color, información con respecto al logotipo, colocación de ilustraciones e instrucciones de fabricación, etc.

El diseño de un objeto debe realizarse respetando normas nacionales e internacionales como: ANSI (American Nacional Standards Institute), ASME (American Society of Mechanical Engineers, ISO (Internacional Standards Organization), STEP (Standard for the Exchange of Product Data), HTML (Hyper Text Markup Laguage), etc. La figura 1 se presenta el proceso lineal general seguido en un proceso de diseño en ingeniería, en las diversas etapas existirá retroalimentaciones.

El refinamiento del diseño es la etapa en que se comienza a trabajar con los sistemas CAD, está formada por tres áreas que se muestran en la figura 2.

El modelado es el proceso de representación de ideas abstractas, palabras y formas a través del empleo ordenado de texto e imágenes simplificadas con el objeto de generar un prototipo digital y comunicar, 


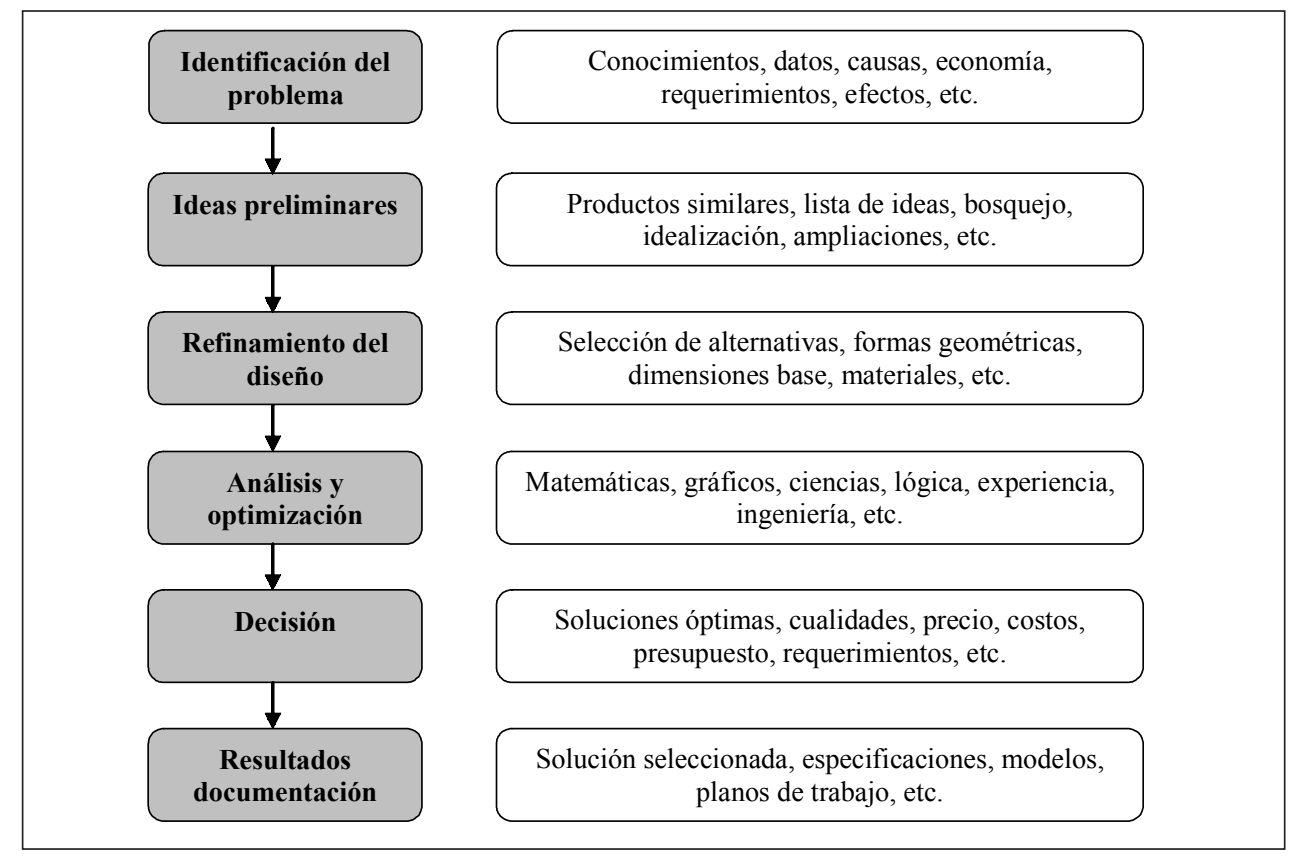

Figura 1. Proceso de diseño

Fuente: Elaboración propia, 2006.

documentar, analizar y visualizar el proceso de diseño. Los tipos de modelo más representativos se presentan en el cuadro 1.

Los modelos tridimensionales paramétricos creados en un sistema de CAD son:

- Modelo de alambre "wireframe" (información geométrica de entrada, usada para la representación de los vértices y aristas).

- De superficie (visualización y trabajos superficiales, usados en control numérico).

- Sólidos (análisis de ingeniería, se le asignan propiedades, son usados en el análisis con elementos finitos).

El modelo debe representar lo mejor posible la realidad, y luego aplicar métodos matemáticos para darle la forma y disposición adecuada para poder obtener resultados numéricos. El diseñador debe determinar si el modelo de computadora satisface o no el criterio de diseño, y debe ser capaz de leer el dibujo, comprender su forma, tamaño e información relativa a su fabricación.

\section{LA INGENIERÍA CONCURRENTE}

La complejidad y constante evolución de la ingeniería ha hecho necesario que las responsabilidades profesionales sean realizadas por gente altamente calificada, con entrenamiento especializado y con diferente formación profesional. El trabajo en equipo no lineal que une los elementos de entrada de proceso y de salida necesarios para elaborar un producto se llama ingeniería concurrente.

Los ingenieros y técnicos deben ser capaces de trabajar en equipo así como diseñar, analizar y comunicarse mediante sistemas CAD. Las gráficas por computadora reúnen a muchos individuos con diferentes necesidades y habilidades visuales; con lo cual se permite que grupos diversos se comuniquen con mayor rapidez y eficiencia.

Dentro del mundo CAD el proceso que permite diseñar un proyecto y compartir toda la información necesaria entre varios componentes de una organización (interna y externa) no importando donde éstos se encuentren es llamado Enterprise Engineering Modeling (EEM - modelado de ingeniería a nivel empresa).

La documentación es el proceso empleado formalmente para registrar y comunicar la solución final de diseño, para ello se puede utilizar una variedad de tipos de dibujos. En el modelo de ingeniería concurrente la información de la base de datos CAD es compartida por muchas áreas de una empresa en especial de aquellas que directamente manipulan dicha información.

\section{INGENIERÍA ASISTIDA POR COMPUTADOR ( CAE)}

CAE simula bajo condiciones aparentemente reales el efecto de variables sobre el elemento diseñado, 


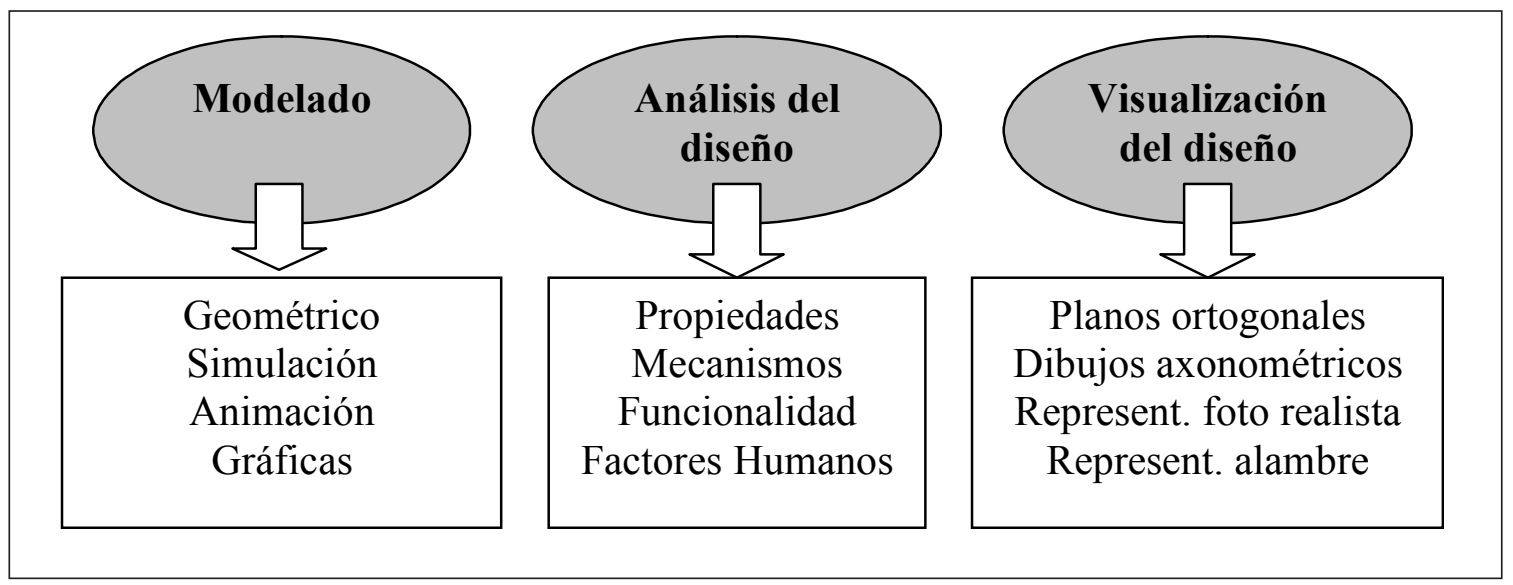

Figura 2. Proceso de refinamiento Fuente: Elaboración propia, 2006.

Cuadro 1. Tipos de modelos computarizados

\begin{tabular}{|l|l|}
\hline Modelo & Características \\
\hline $\begin{array}{l}\text { Geométrico con cortes } \\
\text { virtuales }\end{array}$ & $\begin{array}{l}\text { Representación volumétrica del objeto en el cual se pueden } \\
\text { aplicar rotaciones y secciones. }\end{array}$ \\
\hline $\begin{array}{l}\text { Animación de ensamble y } \\
\text { funcionamiento }\end{array}$ & $\begin{array}{l}\text { Movimiento, tiempo, interferencias y algunas características } \\
\text { generales del sistema propiedad espacio-temporal }\end{array}$ \\
\hline Simulación de procesos & Movimiento, tiempo y las variables importantes del sistema \\
\hline Recorrido virtual & Movimiento, tiempo y escena. \\
\hline Entorno paisajista & $\begin{array}{l}\text { Movimiento, tiempo, luminiscencia, paisaje del entorno, sonidos } \\
\text { naturales. }\end{array}$ \\
\hline Ergonómico & Medidas, formas y movimientos ergonómicos \\
\hline $\begin{array}{l}\text { Aleatoriedad y } \\
\text { transformación }\end{array}$ & Formas, tiempo, movimiento, sonido, transformaciones \\
\hline
\end{tabular}

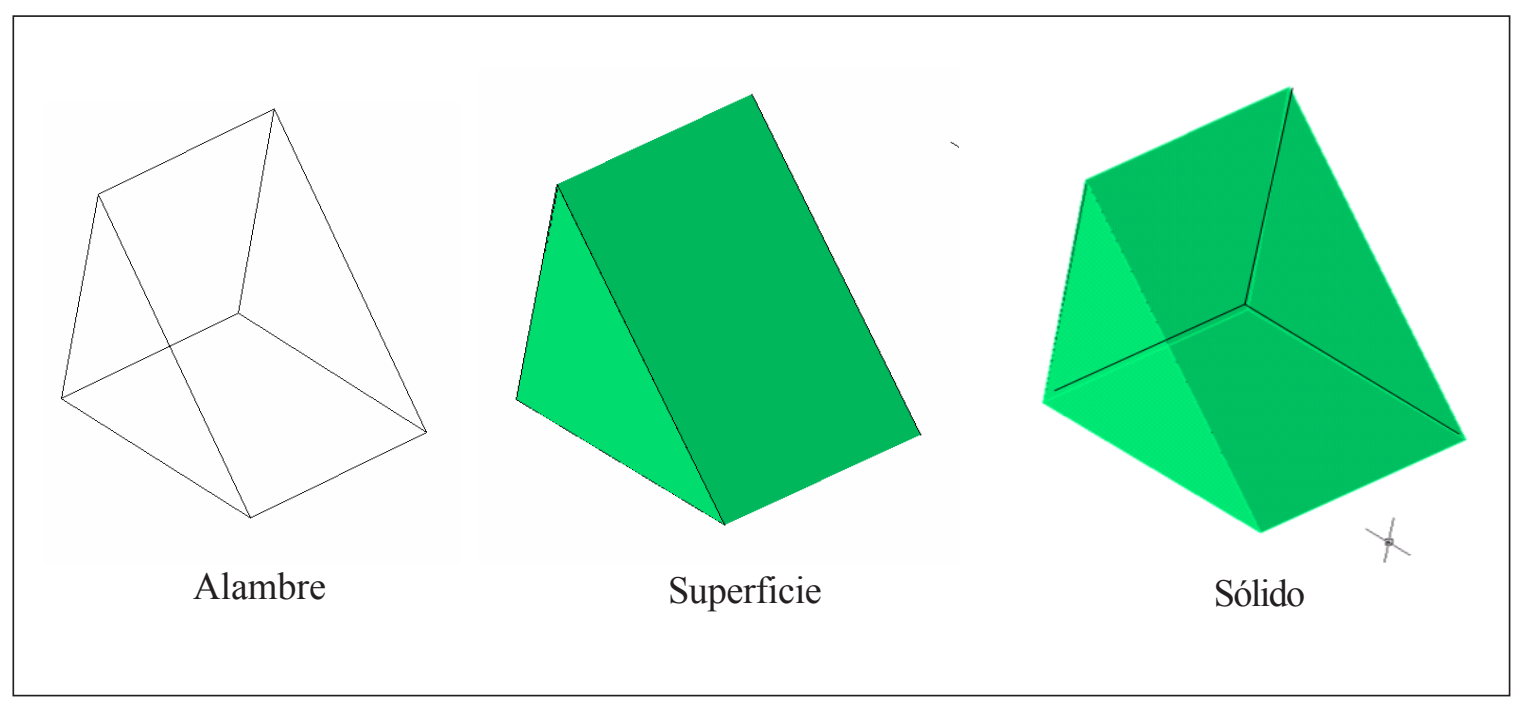




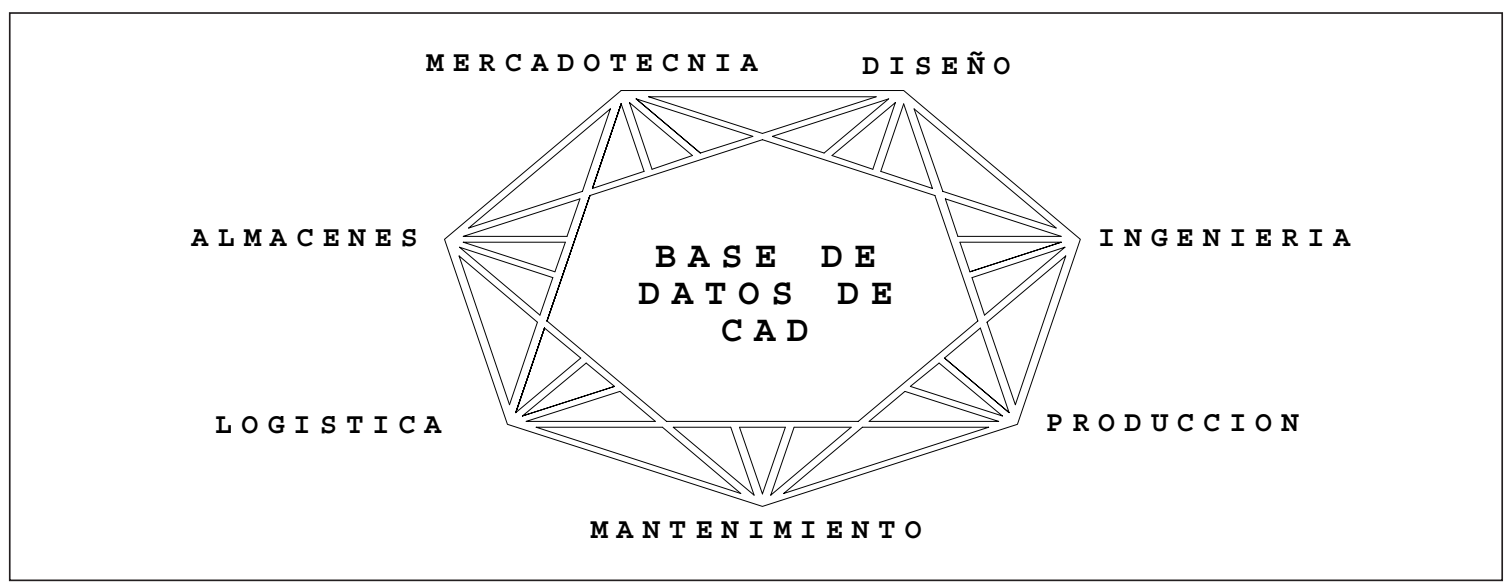

Figura 4. Base de datos CAD

Fuente: Elaboración propia, 2006.

con el fin de llegar a una forma geométrica optimizada para ciertas condiciones. Es un modelado interactivo tridimensional en tiempo real con análisis mediante pruebas no destructivas.

Diseñadores, ingenieros, industriales, arquitectos, etc. utilizan los programas CAE para verificar la factibilidad de distintas alternativas de diseño. Cuando el CAE se utiliza correctamente, se pueden obtener en poco tiempo soluciones eficientes con un alto grado de confianza. La repercusión más importante es que posibilita el diseño mediante ciclos de prueba ya que las primeras informaciones obtenidas por el CAE es sólo la base para la discusión de factibilidad en la que intervendrán la experiencia y la evolución futura.
El análisis por elementos finitos (FEA) ha ayudado a disminuir la cantidad de prototipos necesarios, fijar ciertos valores referenciales de coeficientes de seguridad y analizar distintas opciones de diseño cambiando la geometría o el material en un tiempo reducido; permitiendo que los productos no lleguen tarde al mercado lo que implicaría una pérdida considerable de su ciclo de vida (tiempo en que es rentable su producción).

El FEA está considerado como una de las herramientas más potentes y probadas para la solución de problemas de ingeniería y ciencia aplicada. En muchas ocasiones es de uso indispensable.

Los errores del análisis de los sistemas CAE ocurren comúnmente en la definición del problema y en

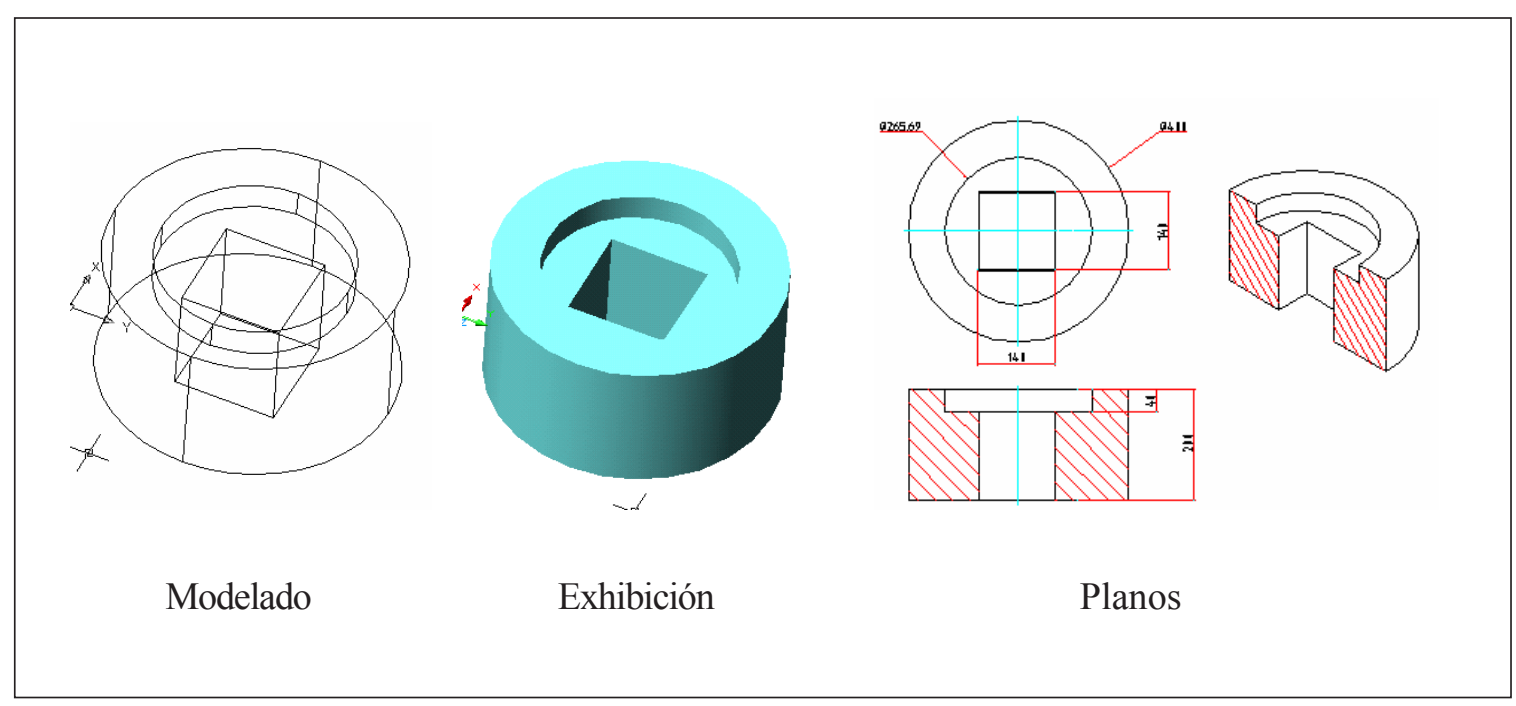

Figura 5. Usos del dibujo asistido por computadora Fuente: Elaboración propia, 2006. 
el uso inapropiado de las condiciones de frontera. Los resultados obtenidos en CAE son aproximados y dependen de cómo se divida la estructura en elementos finitos (el tiempo de resolución del problema se incrementa geométricamente con la cantidad de elementos). También los materiales presentan imperfecciones (fisuras, incrustaciones, rugosidad superficial) y sus propiedades pueden variar por zonas (variaciones de densidad, dureza, distintas orientaciones de los granos en la misma pieza, tensiones remanentes de temple, etc.) lo que puede conllevar a errores no definidos.

Dentro del trabajo CAD, los dibujos asistidos por computador, básicamente son utilizados en la creación de la geometría paramétrica, en la exhibición del sólido y en la generación de los planos ortogonales.

\section{ANÁLISIS POR ELEMENTOS FINITO (FEA)}

Aunque el nombre FEA se ha establecido por los años 1960 , el concepto se ha usado desde hace varios siglos. El empleo de métodos de discretizado espacial y temporal y la aproximación numérica para encontrar soluciones a problemas en ciencia aplicada e ingeniería es conocido desde la antigüedad y en los últimos años muy desarrollado y aprovechado por las industrias aeroespacial y aeronáutica.

Este tipo de cálculo se usó en la construcción de las pirámides egipcias, donde empleaban métodos de discretizado para determinar el volumen de las pirámides. Arquímedes (287 - 212 a. C.) empleaba el mismo método para calcular el volumen de sólidos y de áreas. En la China, Lui Hui (300 d. C.) empleaba un polígono regular de 3072 lados para calcular longitudes de circunferencias con lo que conseguía una aproximación al número Pi de 3.1416.

El concepto básico del análisis por elementos finitos para una estructura radica en sustituir la estructura continua real por una estructura idealizada equivalente compuesta por un número finito de partes discretas (puntos interconectados entre si llamados nodos y al conjunto se le conoce como malla) por medio de un enmallado (bidimensionales; cuadriláteros y triángulos; tridimensionales: hexaedros y tetraedros), al cual se aplican cargas en los nodos de los elementos límite del modelo, después del cual empleando teoremas de energía (relación entre fuerzas actuantes en los nodos y sus desplazamientosley de Hooke, módulo de Young, módulo de Poison, etc.), se llega a derivar una matriz de rigidez (stiffness matriz, - las ecuaciones que rigen el comportamiento de la malla regirán también para la estructura). De esta forma se consigue pasar de un sistema continuo (infinitos grados de libertad), que es regido por un sistema de ecuaciones diferenciales, a un sistema con un número de grados de libertad finito.

El procesador FEA genera una voluminosa cantidad de datos (archivos pesados) los cuales son pos-procesados por las herramientas de visualización de displays gráficos (rendering foto realista con una escala de colores, animaciones, esquemas, etc.) para mostrar esfuerzos internos y con los desplazamientos se obtiene las deformaciones (relacionadas directamente con las tensiones). En la etapa de posproceso se puede ver las magnitudes y localizacio-

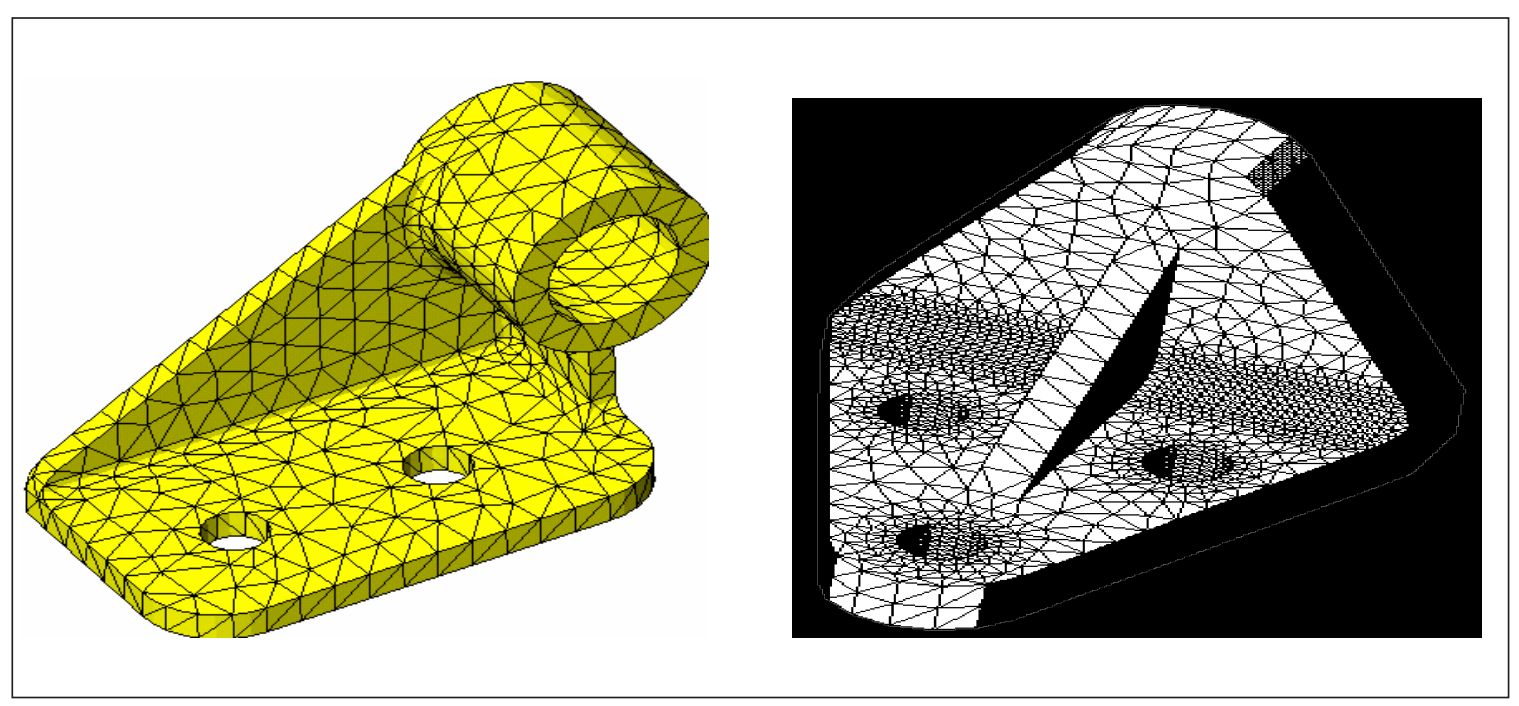

Figura 3. Modelos tridimensionales paramétricos Fuente: Elaboración propia, 2006. 
Cuadro 2. Procedimiento general para el cálculo de elementos finitos

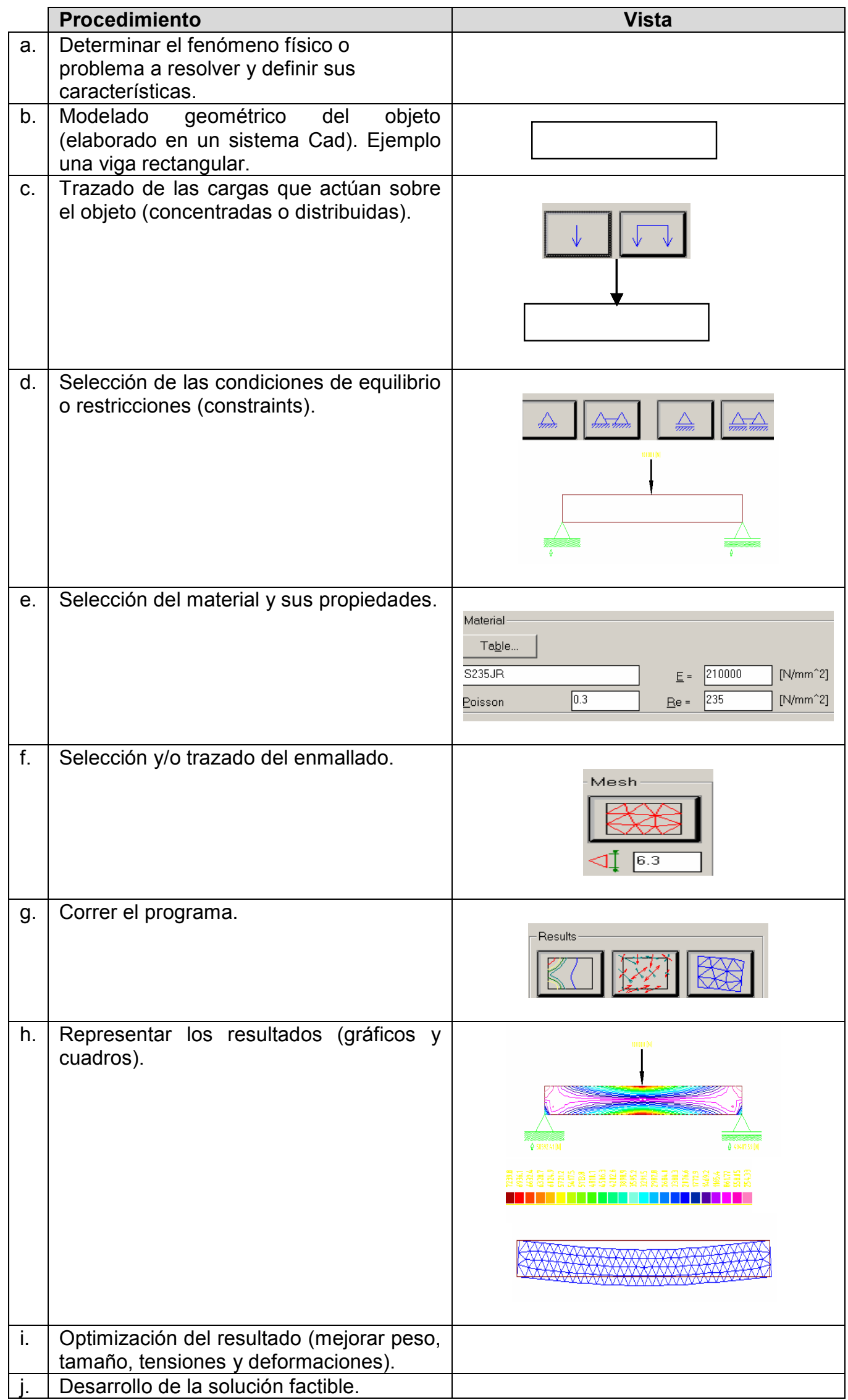


nes de las deformaciones en cualquier instante y en cualquier parte del modelo así como los distintos rasgos de tensión bandas representados por un mismo color lo que permite evaluar rápidamente las zonas críticas del diseño y analizar si la pieza soporta la tensión admisible del material sin deformarse mas de lo permitido por el diseño (zona elástica).

El modelo es más realista cuanto más elementos contengan la malla así como la técnica usada, pero el proceso de cómputo se puede incrementar considerablemente originando una acumulación de errores por redondeo.

EI FEA permite realizar un modelo matemático de cálculo del sistema real, que es más fácil y económico de modificar comparado con el de un prototipo. Los prototipos siguen siendo necesarios, pero en menor número.

Un campo del FEA en pleno desarrollo es el cálculo con cargas dinámicas, vibraciones, impacto y fatiga. Dicho análisis se concentra en evitar que se trabaje con carga cíclicas con frecuencia cercanas a la natural (se podría generar efectos resonantes), así como la falla por fatiga (la pieza se somete a una carga cíclica y se rompe a pesar de que las tensiones, que producen la máxima amplitud de carga, ni siquiera se aproximan a las tensiones de rotura del material utilizado).

El procedimiento general que siguen los software que aplican la técnica de los elementos finitos se presenta en el cuadro 2. Asimismo, en el cuadro 3 se muestran los análisis comunes realizados con esta técnica.

\section{CONCLUSIONES}

El sistema CAD se viene desarrollando en forma acelerada y debido al desarrollo del software y hardware su aplicación se está generalizando tanto en el ámbito académico como empresarial.

En el diseño y análisis de componentes es viable una solución analítica o experimental computarizada a un bajo costo y con alto nivel de confiabilidad. Es así que las técnicas de expresión gráfica se han convertido en el mejor lenguaje para la descripción de objetos.

En el desarrollo de proyectos técnicos se viene usando la técnica de la ingeniería concurrente vía red con geoprocesamiento referenciado.

Las universidades de los países en desarrollo deben adoptar estas tecnologías, por lo que la inclusión de estos temas en la currícula permitirá una formación completa del futuro ingeniero industrial.

Buscar el aprovechamiento de recursos de la UNMSM y construir un laboratorio CAD/CAE con software y hardware apropiados para uso de todas las facultades que lo requieran.

\section{REFERENCIAS BIBLIOGRÁFICAS}

1. Autodesk (2005) http://www.autodesk.com, United States.

2. Bertoline G., Wiebe E., Millar C., y Mohler J. (1999) Dibujo en Ingeniería y Comunicación Gráfica. $2^{\mathrm{a}}$. Ed., McGraw-Hill, México.

Cuadro 3 . Análisis comunes aplicando FEA

\begin{tabular}{|l|l|l|}
\cline { 2 - 3 } \multicolumn{1}{l|}{} & Análisis & Detalle \\
\hline a. & $\begin{array}{l}\text { Propiedades físicas, } \\
\text { térmicas y } \\
\text { mecánicas. }\end{array}$ & $\begin{array}{l}\text { Resistencia, tamaño, volumen, peso, densidad, centro de } \\
\text { gravedad, centro de rotación, transferencia de calor, } \\
\text { conductividad térmica, electromagnetismo, plasticidad, } \\
\text { flujo hidráulico, acústica, etc. }\end{array}$ \\
\hline b. & Mecanismos & $\begin{array}{l}\text { Movimientos, ensambles, interferencias, conexiones, } \\
\text { cargas estáticas y dinámicas, etc. }\end{array}$ \\
\hline c. & Funcional & Cumple con las especificaciones funcionales \\
\hline d. & Factores humanos & $\begin{array}{l}\text { Necesidades físicas, mentales, seguridad, formas y } \\
\text { medidas ergonómicas, etc. }\end{array}$ \\
\hline e. & Estético & $\begin{array}{l}\text { Apariencia y percepción, forma, presentación, color, } \\
\text { textura, etc. }\end{array}$ \\
\hline f. & Mercado & $\begin{array}{l}\text { Preferencias, satisfacción, calidad, precio, presentación, } \\
\text { etc. }\end{array}$ \\
\hline g. & Financiero & Inversiones, presupuesto, costos, precio, etc. \\
\hline
\end{tabular}


3. Cincunegui D. (1999) CADXPress. Ingeniería asistida. 3(15): 20-22Cuellar J.(2000) CADXPress. Ingenieros digitales. 4(25): 36-39.

4. Crear3d (2005)http://www.crear3d.com, Perú.

5. Gould L. (1998) Metalmecánica Internacional. 1(3): 22-25.

6. Estudios y Soluciones Informática de Ingeniería (2005) http://www.esiisl, España.
7. Rohrer M., Strong B. (1998) Metalmecánica Internacional 2(6): 22-29.

8. Semco Cad (agosto, 2005) http:// www.semco.com.pe, Perú.

9. Solidworks Corporation (setiembre, 2005) http:// www.solidworks.com, USA.

10. Trotz L, Poletillo J. (1999) CADXPress. Ingeniería asistida. 3(15): 24-31. 